\title{
A closed-form solution for the seismic racking and rocking behavior of
} rectangular tunnels

\author{
Carlos Gordo-Monsó*, Jesús González-Galindo, Claudio Olalla-Marañón \\ E. T. S. de Ingenieros de Caminos, C. y P., Universidad Politécnica de Madrid, 28040 Madrid, Spain
}

Keywords:

Racking

Rocking

Underground

Tunnel

Seismic

Soil-structure interaction
A B S T R A C T

\begin{abstract}
Current design methodologies for underground structures subjected to earthquake action are based on closedform solutions of the so-called racking coefficient, which relates the soil free-field distortion deformation to the structure distortion deformation. Existent closed-form expressions for this racking coefficient are derived for circular tunnel geometries embedded in an elastic and homogeneous medium, while expressions for rectangular geometries are based on analogies of the circular case. In this paper, a new and intuitive methodology derived from first principles to obtain a closed-form expression for the racking coefficient for rectangular-like tunnel geometries buried at a sufficient depth is presented, and it is shown that it provides a good fit to finite element analyses results. Similarly, a procedure to obtain closed-form expressions for the rocking rotation of the structure, and the racking deformation profile at different distances from the soil-tunnel interface is presented.
\end{abstract}

\section{Introduction}

Underground structures behavior subjected to the earthquake action differ notably from the case of above ground structures. In most cases for underground structures, the inertial component of the structure mass displacement is negligible and does not govern the dynamic behavior during an earthquake (Bobet, 2010; Huo et al., 2006; Penzien, 2000; Pitilakis et al., 2007; Tsinidis et al., 2015a; Wang, 1993), whereas for surface structures this is the most significant parameter. Moreover, buried structures are constrained by the surrounding soil, and hence subjected largely to a displacement range similar to that undergone by the soil (Bobet et al., 2008; Hashash et al., 2010; Huo et al., 2005).

To assess the displacements, and hence internal forces, of underground structures during an earthquake, three different approaches are followed (Bobet et al., 2008; Hung et al., 2009; LA Metro Authority, 2012; WSDOT, 2010), these are: pseudo-static free-field displacement approaches, pseudo-static soil-structure interaction displacement approaches, and numerical soil-structure interaction approaches.

Pseudo-static free-field displacement approaches (Hendron and Fernandez, 1983, Merrit et al., 1985, Monsees, 1991) constitute the early attempts to study the linked behavior of soil and underground structure during an earthquake. The first proposed method, developed for the San Francisco Trans-Bay Tunnel (Kuesel, 1969), accurately describes the main features of the problem: regarding the soil, the earthquake consists of a set of passing deformation waves that the embedded underground structure must accommodate with limited damage. The approach consists, at a first stage, in determining the soil displacement profile, at a distance far enough of the structure (i.e. freefield displacement), and at the depth comprised by the underground structure (i.e. depth between invert slab and roofing system). A second stage consists in imposing this free-field displacement to the structure, analyze the internal forces, and design structural elements accordingly. Given that this approach imposes a free-field displacement to the structure, it does not consider that the soil-structure interaction may locally change the deformation profile in the nearby soil surrounding the underground structure.

Recognizing that the embedded structure presence may alter the soil deformation pattern in its proximity, a more refined approach termed pseudo-static soil-structure interaction method, was developed during the 90 s by several authors, for which a comprehensive review is provided by Hashash et al. (2001). Similar in concept to the free-field displacement approach, once that the soil displacement profile is determined, an amplification or reduction factor termed racking coefficient is applied to the free-field deformation to obtain the structure deformation which must be accommodated with limited damage. This racking coefficient, which encapsulates the soil-structure interaction (SSI) effect, was obtained analytically based on the elasticity theory, and further verified by numerical analyses. This method has been substantiated in different closed-form expressions for the racking coefficient, each one developed under slightly different assumptions.

\footnotetext{
* Corresponding author.

E-mail addresses: carlos.gordom@upm.es (C. Gordo-Monsó), jesus.gonzalezg@upm.es (J. González-Galindo), claudio.olalla@upm.es (C. Olalla-Marañón).
} 


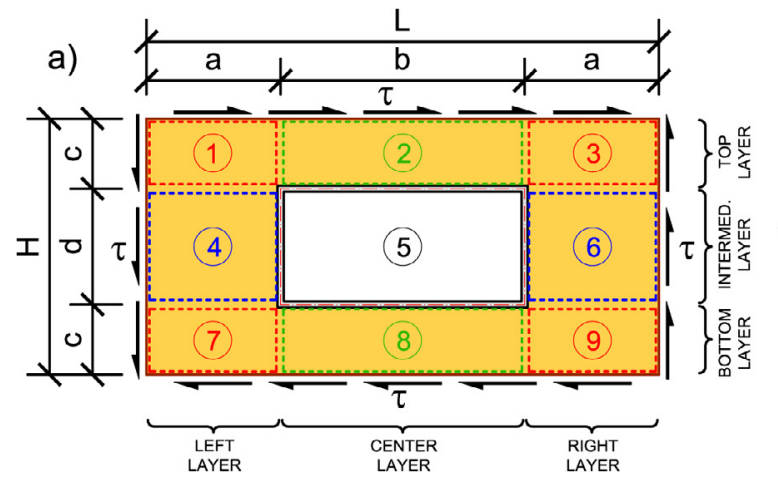

b)

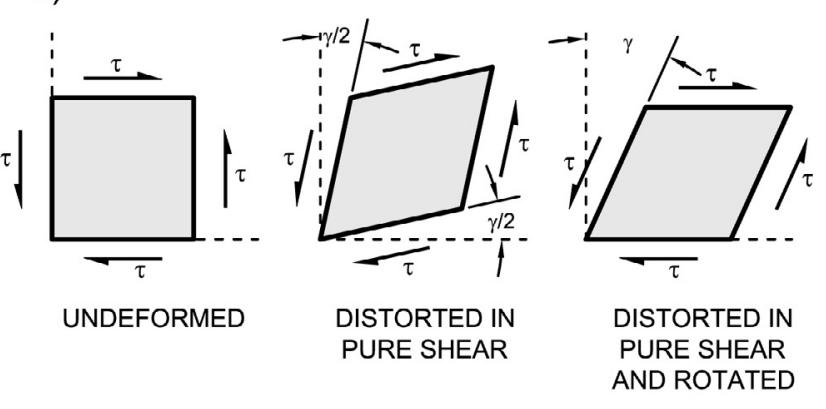

Fig. 1. (a) Block diagram of an arbitrary portion of the soil-structure system. (b) Pure shear distortion strain scheme for an individual block.

Wang (1993) proposed that a single parameter $F_{R}$ termed flexibility ratio (measuring the relative shear stiffness of the soil substituted by the underground structure, compared to the shear stiffness of the structure itself) governs the SSI effect (the more flexible the structure is respective to the soil, the more the structure deforms and vice versa), and proposed a closed form expression for the rocking coefficient of circular tunnels based on previous work by Peck (1969). Penzien (2000), Penzien and Wu (1998) developed a background for the racking coefficient based on the theory of elasticity and provided a closed-form solution for the racking coefficient similar to that of Wang. Anderson et al. (2008) disregard the effect that the Poisson coefficient may have in the SSI phenomenon, providing a closed-form expression similar to that of Wang and Penzien corresponding to a constant Poisson coefficient $\nu=0.5$. Huo et al. (2006) acknowledge that the previously obtained racking coefficient expressions for rectangular tunnels disregard the effect of the out-of-plane displacement of the walls and slabs that react against the surrounding soil, and hence develops a closed-form expression for the racking coefficient by means of conformal mapping techniques of a rectangular tunnel and a circular tunnel.

Numerical soil-structure interaction approaches constitute the most detailed methodology to study the effect of an underground structure subjected to the seismic action. These consist on the numerical analysis of the problem by developing a finite elements or finite differences model of the buried structure-soil system, and performing whether a full dynamic analysis or a pseudo-static analysis, both of these linear or non-linear, depending on the feature of interest to be studied (Abate and Massimino, 2017; Debiasi et al., 2013; Fabozzi et al., 2017; Guoxing et al., 2015; Hashash et al., 2001; Hung et al., 2009; LA Metro Authority, 2012; Wang et al., 2013; WSDOT, 2010). Although this approach might appear the most thorough and precise, given that it may appropriately consider different mechanical effects, in practice it is seldom used during early design stages of an underground structure (LA Metro Authority, 2012). Firstly, the design process covers conceptual, preliminary, and detailed design phases, during which the functional and architectural planning (i.e. passenger circulations in subway stations) may greatly and frequently alter the previous structural and geotechnical configuration. This rends a detailed finite element approach highly impractical until later design stages, when many relevant engineering decisions may have already been taken, and there is little place for changes but for verification. Secondly, the solely reliance in a complex numerical model for obtaining relevant structural results (i.e. bending moments, shear forces, displacements, etc.) may leave the engineer without a practical and more immediate tool to intuitively guide her decisions towards a safe and economic design.

\section{Purpose of the proposed solution}

As exposed in previous sections, from the closed-form expressions available to date for the analysis of the SSI effect, and hence the racking deformation of a rectangular underground structure, 3 of them
(Anderson et al., 2008; Penzien, 2000; Wang, 1993) rely on the assumption that the stress and strain field in the surrounding soil is similar to the one developed for a circular cross-section. The expression by Huo et al. (2006) is effectively derived for a rectangular cross section, nevertheless the underlying mathematical development is intricate and may hinder the understanding of the mechanical phenomena to the engineer designing the underground structure.

In the following, we propose a closed-form solution developed for the rectangular geometry case that, while trying to be as accurate as possible, provides the engineer with an effective mean to intuitively understand the very nature of the racking effect (namely, a larger structure deformation than the one corresponding to the free-field if the structure is more flexible than the soil, and vice versa). This intuitive understanding is an aspect that should not be disregarded: as explained before, the free field methods (Kuesel, 1969; Monsees, 1991) are based on the assumption that the soil close to the embedded structure deforms similarly to the soil far from it and that no SSI effect is present, probably guided by an intuition that was corrected with later studies.

\section{Proposed solution for the racking coefficient}

Consider a rectangular underground structure (of width $b$, depth $d$, and aspect ratio $\lambda=b / d$ ), and a portion of surrounding soil of arbitrary size (of width $L$ and depth $H$ ) concentric with the underground structure as in Fig. 1a. Consider that the soil exterior perimeter is subjected to a pure shear stress state, and consider that our purpose is to determine the strain field in the soil and structure.

In determining the strain field, the following 3 assumptions are made:

- The soil-structure system can be divided in rectangular blocks, numbered 1-9 (Fig. 1a).

- Each block can only deform in a pure shear pattern (Fig. 1b), with the shear stiffness of an elastic, homogenous, and isotropic medium, defined by its shear modulus $G$ and the elasticity equation $\gamma=\tau / G$. Note that this equation measures the total sheared angle $\gamma$ between orthogonal boundaries, but that the sheared angle respective to the vertical and horizontal boundaries of the block is $\gamma / 2$ each (Hudson and Harrison, 2000).

- There is displacement compatibility between corners of adjacent blocks, that is, corners shared by different blocks displace horizontally and vertically the same amount. This would translate to a nonslip condition in terms of previous studies (Penzien, 2000; Wang, 1993).

Stemming from the above, it is assumed that within each block shear stresses and strains are constant through the entire width or depth, in particular at its boundaries. This assumption means that no stress or strain concentration will take place in the corners of the soilstructure, and that only an "average stress" and "average strain" will be 
a)

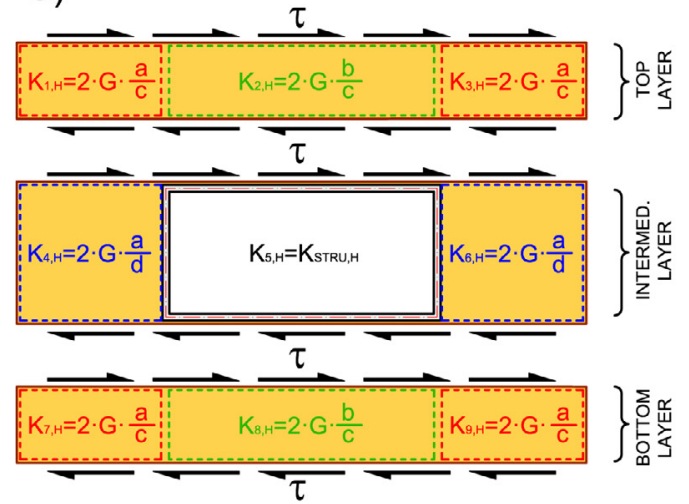

b)

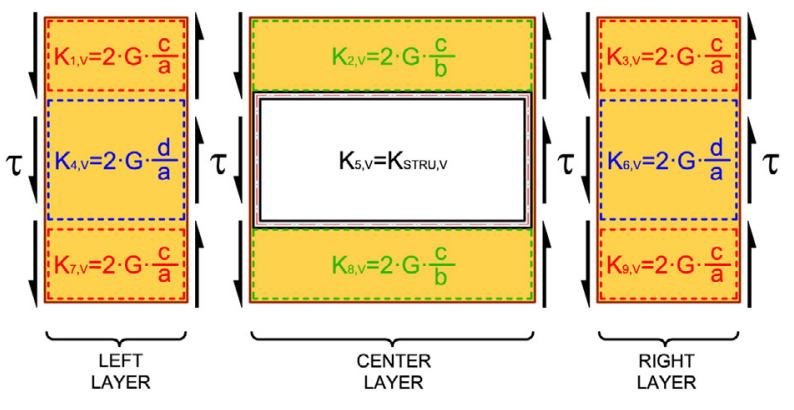

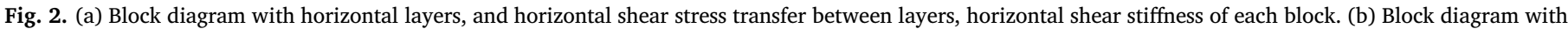
vertical layers, vertical shear stress transfer between layers, and vertical shear stiffness of each block.

considered for each block. Moreover, the total angle $\gamma$ sheared by each block will be the sum of the sheared angle that produces horizontal displacement $\gamma_{H}$, plus the sheared angle that produces vertical displacement $\gamma_{V}$.

If one follows the transfer of, say, horizontal shear force from the top to the bottom boundary through the depth of the system, from equilibrium considerations, it is easy to note that the total shear force at any horizontal section must be constant (Fig. 2a) (even if the horizontal shear stress at each block may be different). Similarly, stemming from the above assumptions, and from displacement compatibility considerations between contiguous blocks, one can note that for each horizontally contiguous block (that is pertaining to the same horizontal layer, namely the top layer of blocks $1-3$, the intermediate layer of blocks 4-6, and the bottom layer of blocks 7-9) the horizontal shear distortion strain $\gamma_{H}$ must be of equal value.

Therefore, the horizontal displacement stiffness for each horizontal layer of blocks must be the in parallel sum of the shear stiffness of each of the three blocks within the layer. In particular, and considering that the horizontal displacement corresponds solely to the horizontal sheared angle $\gamma / 2$, the stiffness $K_{n, H}$ of each block $n$ for a horizontal force will be as shown in Eqs. (1)-(4).

$K_{1, H}=K_{3, H}=K_{7, H}=K_{9, H}=2 \cdot G \cdot \frac{a}{c}$

$K_{2, H}=K_{8, H}=2 \cdot G \cdot \frac{b}{c}$

$K_{4, H}=K_{6, H}=2 \cdot G \cdot \frac{a}{d}$

$K_{5, H}=K_{S T R U, H}=2 \cdot \frac{G \cdot \frac{b}{d}}{F_{R}}$

where the term $F_{R}$ in Eq. (4) corresponds to the classical flexibility ratio as defined by Wang (1993), which in the case of linearly elastic a single barrel rectangular tunnel of width $b$, depth $d$, and wall and slab bending inertias $I_{w}$ and $I_{b}$ respectively is defined by Eq. (5).

$F_{R}=\frac{K_{S O I L}}{K_{S T R U}}=\frac{G \cdot \frac{b}{d}}{\frac{1}{\frac{d^{2} \cdot b}{24 \cdot E \cdot I_{b}}+\frac{d^{3}}{24 \cdot E \cdot I_{w}}}}$

Note that this particular expression for $F_{R}$ is not relevant for the procedure described in this paper, and can be easily changed to any other shear deformation stiffness for a given application (i.e. stiffness with inner columns, inner horizontal slabs, etc.).

A similar reasoning would be valid for the vertical shear force transferring right to left (Fig. 2b). Using appropriate geometrical dimensions for the stiffness $K_{n, V}$ of each block $n$ for a vertical force will be as shown in Eqs. (6)-(9).

$K_{1, V}=K_{3, V}=K_{7, V}=K_{9, V}=2 \cdot G \cdot \frac{c}{a}$

$K_{2, V}=K_{8, V}=2 \cdot G \cdot \frac{c}{b}$

$K_{4, V}=K_{6, V}=2 \cdot G \cdot \frac{d}{a}$

$K_{5, V}=K_{S T R U, V}=2 \cdot \frac{G \cdot \frac{d}{b}}{F_{R}}$

From the previous expressions (1)-(4), and owing to the shear strain compatibility between horizontal adjacent blocks, the shear stiffness for each horizontal layer ( $K_{T O P}$ for the top layer, $K_{I N T}$ for the intermediate, and $K_{B O T}$ for the bottom layer) can be obtained as an in-parallel sum of stiffness (Eqs. (10) and (11)).

$$
\begin{aligned}
K_{T O P, H} & =K_{B O T, H}=K_{1, H}+K_{2, H}+K_{3, H}=K_{7, H}+K_{8, H}+K_{9, H} \\
& =2 \cdot G \cdot \frac{a+b+a}{c}=2 \cdot G \cdot \frac{L}{c} \\
K_{I N T, H} & =K_{4, H}+K_{5, H}+K_{6, H}=2 \cdot G \cdot \frac{2 a}{d}+K_{S T R U, H}
\end{aligned}
$$

The shear stiffness for each vertical layer $\left(K_{L E F T}\right.$ for the left layer, $K_{C E N T}$ for the central layer, and $K_{R I G H T}$ for the right layer) can be obtained similarly (Eqs. (12) and (13)).

$$
\begin{aligned}
K_{L E F T, V} & =K_{R I G H T, V}=K_{1, V}+K_{4, V}+K_{7, V}=K_{3, V}+K_{5, V}+K_{9, V} \\
& =2 \cdot G \cdot \frac{c+d+c}{a}=2 \cdot G \cdot \frac{H}{a}
\end{aligned}
$$

$K_{C E N T, V}=K_{2, V}+K_{5, V}+K_{8, H}=2 \cdot G \cdot \frac{2 c}{b}+K_{S T R U, V}$

If one were to estimate the average horizontal shear stiffness $\mathrm{K}_{\mathrm{AVG}, \mathrm{H}}$ of the soil and structure ensemble (that is the 9 blocks), by measuring the force required at the top face in order to produce a unit horizontal displacement, an appropriate method consistent with the above assumptions would be to sum the in-series stiffness of each horizontal layer (Eq. (14)).

$K_{A V G, H}=\frac{1}{\frac{1}{K_{T O P, H}}+\frac{1}{K_{I N T, H}}+\frac{1}{K_{B O T, H}}}=\frac{1}{2 \cdot \frac{1}{2 \cdot G \cdot \frac{L}{c}}+\frac{1}{2 \cdot G \cdot \frac{2 a}{d}+K_{S T R U, H}}}$

Similarly for the average vertical shear stiffness $K_{A V G, V}$ of the soil and structure ensemble, adding the in-series stiffness of each vertical layer (Eq. (15)). 
$K_{A V G, V}=\frac{1}{\frac{1}{K_{L E F T, V}}+\frac{1}{K_{C E N T, V}}+\frac{1}{K_{R I G H T, V}}}=\frac{1}{2 \cdot \frac{1}{2 \cdot G \cdot \frac{H}{a}}+\frac{1}{2 \cdot G \cdot \frac{2 c}{b}+K_{S T R U, V}}}$

With the previous results at hand, one can compute an approximate racking coefficient for the shear distortion of the embedded structure and the average shear distortion of the soil and structure ensemble, that is to say, the ratio of shear distortions between the inner structure $\gamma_{I N N}=\gamma_{I N T, H}+\gamma_{C E N T, V}$ and the outer perimeter $\gamma_{A V G}=\gamma_{A V G, H}+\gamma_{A V G, V}$, when the outer perimeter is subjected to a pure shear stress state of value $\tau$. This racking coefficient would be the ratio of the sum of strains as shown in Eq. (16), where the hat in $\widetilde{R}$ denotes that this racking expression is approximate and conditional to the block behavior assumptions stated before.

$\widetilde{R}=\frac{\gamma_{I N T, H}+\gamma_{C E N T, V}}{\gamma_{A V G, H}+\gamma_{A V G, V}}=\frac{\frac{\tau \cdot L / K_{I N T, H}}{d}+\frac{\tau \cdot H / K_{C E N T, V}}{b}}{\frac{\tau \cdot L / K_{A V G, H}}{H}+\frac{\tau \cdot H / K_{A V G, V}}{L}}=\frac{\frac{L}{d \cdot K_{I N T, H}}+\frac{H}{b \cdot K_{C E N T, V}}}{\frac{L}{H \cdot K_{A V G, H}}+\frac{H}{L \cdot K_{A V G, V}}}$

From the previous expressions, and for the sake of simplicity considering only the horizontal shear strain contribution (Eqs. (10), (11), (14), and (16)), one can readily obtain some important information regarding the behavior of the soil and structure ensemble. As it would be expected, if the intermediate layer were stiffer than the soil-structure ensemble, the intermediate layer would deform less than the ensemble (Fig. 3a). On the contrary, if the intermediate layer were softer, the intermediate layer would deform more than the soil and structure ensemble (Fig. 3b).

Notice that the distortion stiffness of the intermediate layer is governed by the distortion stiffness of the embedded structure: a small structure stiffness when compared with the substituted soil $\left(F_{R}>1\right)$ would yield an intermediate layer softer than the ensemble, and vice versa. A similar reasoning can be made regarding the vertical component of the shear strain, for which two cases one with a stiffer structure than soil $\left(F_{R}<1\right)$, and another with a softer structure than soil $\left(F_{R}>1\right)$ are depicted in (Fig. 4a) and (Fig. 4b) respectively.

Until this point in the discussion, the dimensions $L$ and $H$ for the soil perimeter have been chosen arbitrarily. If we think for which dimensions the block assumptions may be approximately valid, we may consider that, for a soil perimeter close enough to the structure, the average shear distortion can be supposed constant in each layer. Therefore, we may think that we can compute approximately a racking coefficient between a soil perimeter close enough to the structure and the structure itself by means of Eq. (16).

Moreover, we may assume that if we consider successive soil control perimeters (Fig. 5), close enough one to each other (say perimeters $i-$ 1 and $i$ ), we may compute an approximate individual racking coefficient $\widetilde{R_{i}}$, relating the shear distortion of successive perimeters, using Eqs. (14)-(16).

For doing so in any given control perimeter $i$, it will suffice to substitute the structure stiffness $K_{S T R U, H}$ and $K_{S T R U, V}$ in Eqs. (14) and

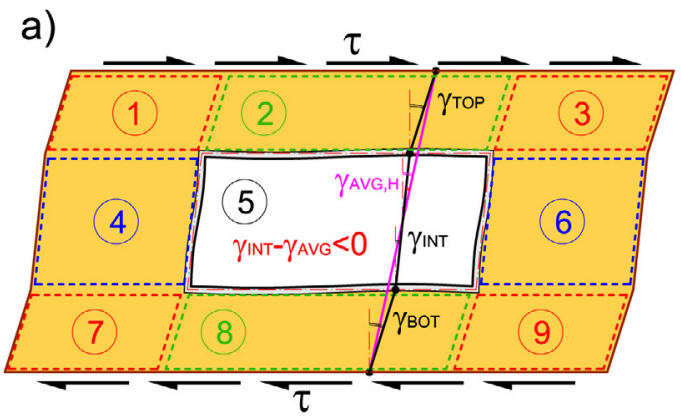

(15) with the average horizontal and vertical stiffness of the more internal perimeter $i-1, K_{A V G, H, i-1}$ and $K_{A V G, V, i-1}$, and choose geometrical dimensions $a_{i}, b_{i}, c_{i}, d_{i}, L_{i}$ and $H_{i}$ corresponding to the given control perimeter $i$, as shown in Eq. (17).

$\tilde{R}_{i}=\frac{\gamma_{A V G, H, i-1}+\gamma_{A V G, V, i-1}}{\gamma_{A V G, H, i}+\gamma_{A V G, V, i}}=\frac{\frac{L_{i}}{d_{i} \cdot K_{I N T, H, i-1}}+\frac{H_{i}}{b_{i} \cdot K_{C E N T, V, i-1}}}{\frac{L_{i}}{H_{i} \cdot K_{A V G, H, i}}+\frac{H_{i}}{L_{i} \cdot K_{A V G, V, i}}}$

If we follow the reasoning, we may think that the total racking coefficient relating the embedded structure shear distortion $\gamma_{S T R U}$, and the free-field distortion $\gamma_{F F}$, can be approximated by a sufficiently large portion of soil surrounding the structure with a fine discretization of successive control perimeters, and by the product of each individual racking coefficient as in the multiplicative Eq. (18).

$R=\frac{\gamma_{S T R U}}{\gamma_{F F}} \cong \prod_{i=1}^{i=n} \frac{\gamma_{i-1}}{\gamma_{i}}=\prod_{i=1}^{i=n} \widetilde{R}_{i}$

In Eq. (18) the $\widetilde{R_{i}}$ expression is given by Eq. (17), and in Eq. (17) for $i=1$ corresponding to the innermost control perimeter, the $K_{A V G, H, i-1}=0$ and $K_{A V G, V, i-1=o}$ are the structure stiffness given by Eqs. (4) and (9).

From the exposition above, we must note that the proposed pure shear block model cannot consider appropriately the solution dependence on the Poisson coefficient demonstrated by other researchers (Huo et al., 2006; Penzien, 2000; Wang, 1993). This is because, being each block subjected to a pure shear state, there is no direct dependence for the shear strain on the Poisson coefficient once that the $G$ modulus is defined. Given that in a pure shear state there is no change in volume, one might expect that the proposed multiplicative closed-form solution should best replicate the behavior for those cases in which the Poisson coefficient is close to $\nu=0.5$. This lack of dependence of the proposed model on the Poisson coefficient can be adjusted introducing a modification factor which will be presented later in this paper.

In order to verify the accuracy of the proposed closed-form solution for the racking coefficient, a series of finite element models (FEM) have been carried out in OpenSEES (McKenna et al., 2010). The FEM analyses developed represent the problem of a sufficiently large elastic, isotropic, and homogenous soil medium with an embedded rectangular structure at its center. The soil has been meshed with 4 nodes quadrilateral elements and plane-strain behavior, whereas the lining meshing has been performed with beam elements whose nodes are linked in vertical and horizontal displacement to the adjacent soil nodes, as to represent a fully bonded concrete-soil behavior. The soil external boundaries do not present displacement restrictions, and are subjected to a pure shear stress state of value $\tau$. Only the bottom leftmost soil node has horizontal $\mathrm{u}_{\mathrm{x}}$ and vertical $\mathrm{u}_{\mathrm{y}}$ displacements restricted, and the bottom rightmost soil node has vertical displacement $\mathrm{u}_{\mathrm{y}}$ restricted, as to achieve numerical equilibrium. The considered soil shear wave velocity is $V s=360 \mathrm{~m} / \mathrm{s}$ and the soil specific weight is $\rho=20 \mathrm{kN} / \mathrm{m}^{3}$, yielding a shear modulus $G=259.20 \mathrm{MPa}$. Regarding the tunnel, a concrete structure has been considered with Young

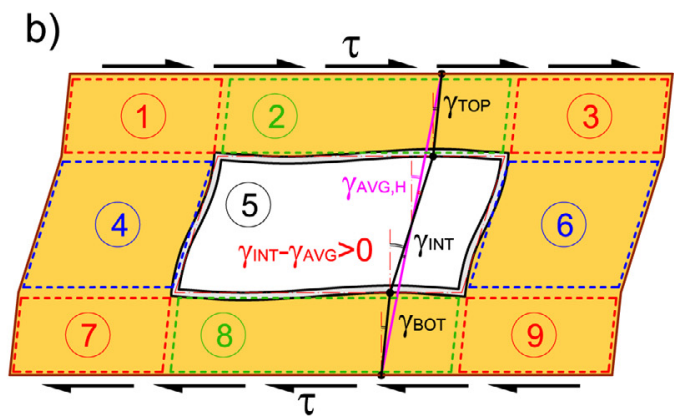

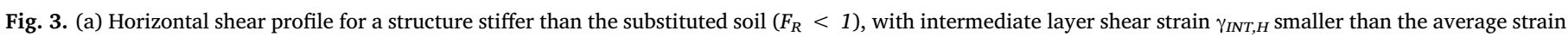

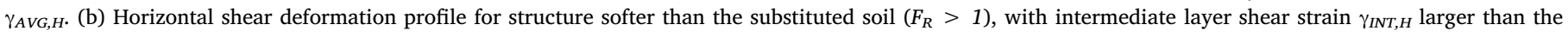
average strain $\gamma_{A V G, H}$. 

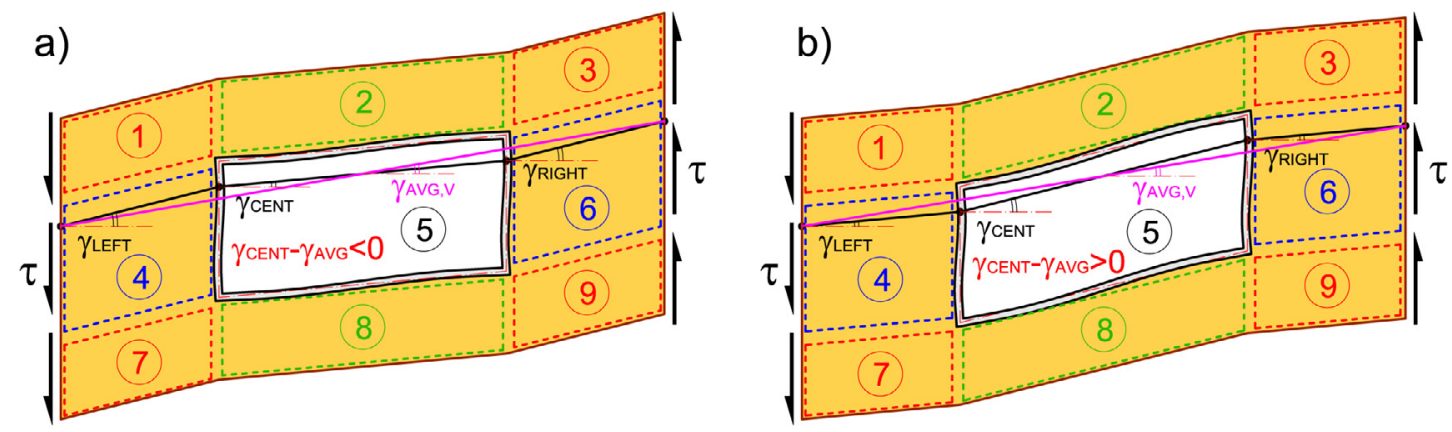

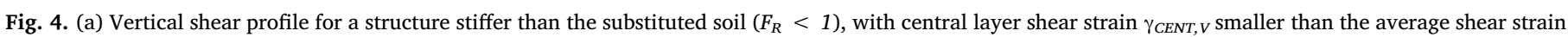

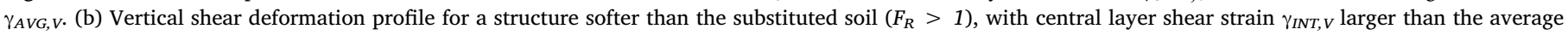
shear strain $\gamma_{A V G, V}$.

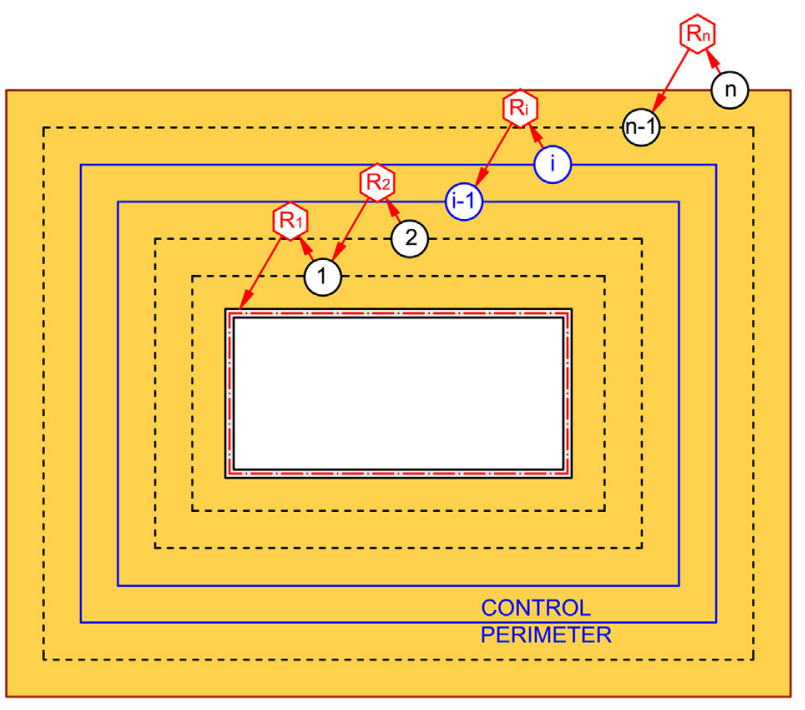

Fig. 5. Successive series of control perimeters 1 to $n$. Each individual racking coefficient $\widetilde{R}_{i}$ measures the ratio of shear distortion between control perimeter $i-1$ and $i$, being $\widetilde{R_{i}}=\frac{\gamma_{A V G, i-1}}{\gamma_{A V G, i}}$.

modulus $E_{c}=30 \mathrm{GPa}$, and constant wall and slab thickness of $h_{w}=h_{b}=1.50 \mathrm{~m}$ providing the gross bending inertia. A range of flexibility ratios $F_{R}$ has been studied by varying the width $b$ and depth $d$ dimensions of the structure from $1 \mathrm{~m}$ to $20 \mathrm{~m}$. Different structure aspect ratios $\lambda=b / d$ ranging from 1 to 3 have been analyzed to study its influence on the solution. The soil boundaries, both for the FEM analyses and the closed form solutions proposed in this paper have been chosen $50 \mathrm{~m}$ away horizontally and vertically from the structure. For the evaluation of the closed-form solution, a value of 1000 control perimeters ( $n=1000$ terms in Eq. (18)) has been employed.

Fig. 6a shows a very good agreement of the proposed multiplicative solution Eq. (18) with the FEM analyses for all the studied aspect ratios $\lambda$, and for the case in which the Poisson coefficient is $\nu=0.5$ as expected. Similarly, this expression closely matches results from other closed-form expressions for circular geometries (Anderson et al., 2008; Penzien, 2000; Wang, 1993) when $\nu=0.5$. For other Poisson coefficient $\nu$ values, and when the modification factor introduced later in this paper is applied, similar good agreement with the FEM analyses, and similar trends as those in other closed-form expressions can be appreciated.

\section{Proposed solution for the racking profile}

We might also consider to analyze and compare the profile of the racking coefficient at different distances (close and far from the structure) along a section as shown in (Fig. 7b), that is, how much an intermediate perimeter distorts respective to the free-field distortion. Regarding the multiplicative closed-form method presented in the previous section, an expression similar to Eq. (18) to compute such profile for a given perimeter $j$ would be Eq. (19).

$R_{\text {PERIMj }}=\frac{\gamma_{\text {PERIMj }}}{\gamma_{F F}} \cong \prod_{i=j+1}^{i=n} \frac{\gamma_{i-1}}{\gamma_{i}}=\prod_{i=j+1}^{i=n} \tilde{R}_{i}$

As for the racking coefficient, the same set of FEM analyses has been used to obtain numerical results for the racking profile. The average shear distortion $\gamma_{\text {FEM,PERIM } j}$ at each control perimeter $j$ in the FEM analyses has been computed as the horizontal and vertical displacement difference for the bottom leftmost corner and the top rightmost corner of each perimeter with Eq. (20) (Fig. 7a), and then normalized by the free-field distortion $\gamma_{F F}$ to obtain the racking profile at each perimeter $j$.

$\gamma_{\text {FEM }, \text { PERIM } j}=\frac{\Delta_{\text {HORIZ }, T O P, j}-\Delta_{\text {HORIZ,BOTTOM }, j}}{H_{j}}+\frac{\Delta_{V E R T, R I G H T, j}-\Delta_{V E R T, L E F T, j}}{L_{j}}$

Results are shown in (Fig. 8) where the horizontal abscissa has been normalized among models as the ratio of the distance of the control perimeter to the structure $X$, divided by the structure width $b$ ( $X_{P E R I M-}$ ${ }_{\text {ETER }} / b_{\text {STRUCTURE }}=0$ means a perimeter adjacent to the structure, and $X_{\text {PERIMETER }} / b_{\text {STRUCTURE }}=2$ means a perimeter at two widths from the structure).

In Fig. 8 the multiplicative expression Eq. (19) shows a good agreement with the finite element results, and indicates that at a distance of 1.5-2 structure widths the shear distortion value remains close to the free-field distortion ( $R \approx 1$ ), that is to say, at farther distances than two widths the influence of the embedded structure becomes almost negligible.

\section{Proposed solution for the rocking coefficient}

Several researchers (Cilingir and Madabhushi, 2011; Debiasi et al., 2013; Iwatate et al., 2000; Pitilakis and Tsinidis, 2016; Tsinidis, 2017; Tsinidis et al., 2015b; Tsinidis and Pitilakis, 2018; Ulgen et al., 2015) have pointed an interesting rocking behavior shown by buried structures presenting relative stiffness departing from the substituted soil stiffness. Stiff structures $\left(F_{R}<1\right)$ tend to present a rigid-body rocking displacement pattern, in the sense that the structure tends to rotate rigidly in the same direction as the global shear distortion. On the contrary, flexible structures $\left(F_{R}>1\right)$ tend to present a rocking pattern in the opposite direction to the global shear distortion. The rocking rotation coefficient is defined by Tsinidis (2017) as the rotated angle $\theta$ by the bottom or top slab, and further normalized by the free field distortion angle as the magnitude $\theta / \gamma_{F F}$. A close-up of the tunnel region and corresponding displacement patterns resulting from FEM analyses is shown in (Fig. 9), similar to the ones by Tsinidis and Pitilakis (Pitilakis and Tsinidis, 2016; Tsinidis, 2017; Tsinidis et al., 2016; 

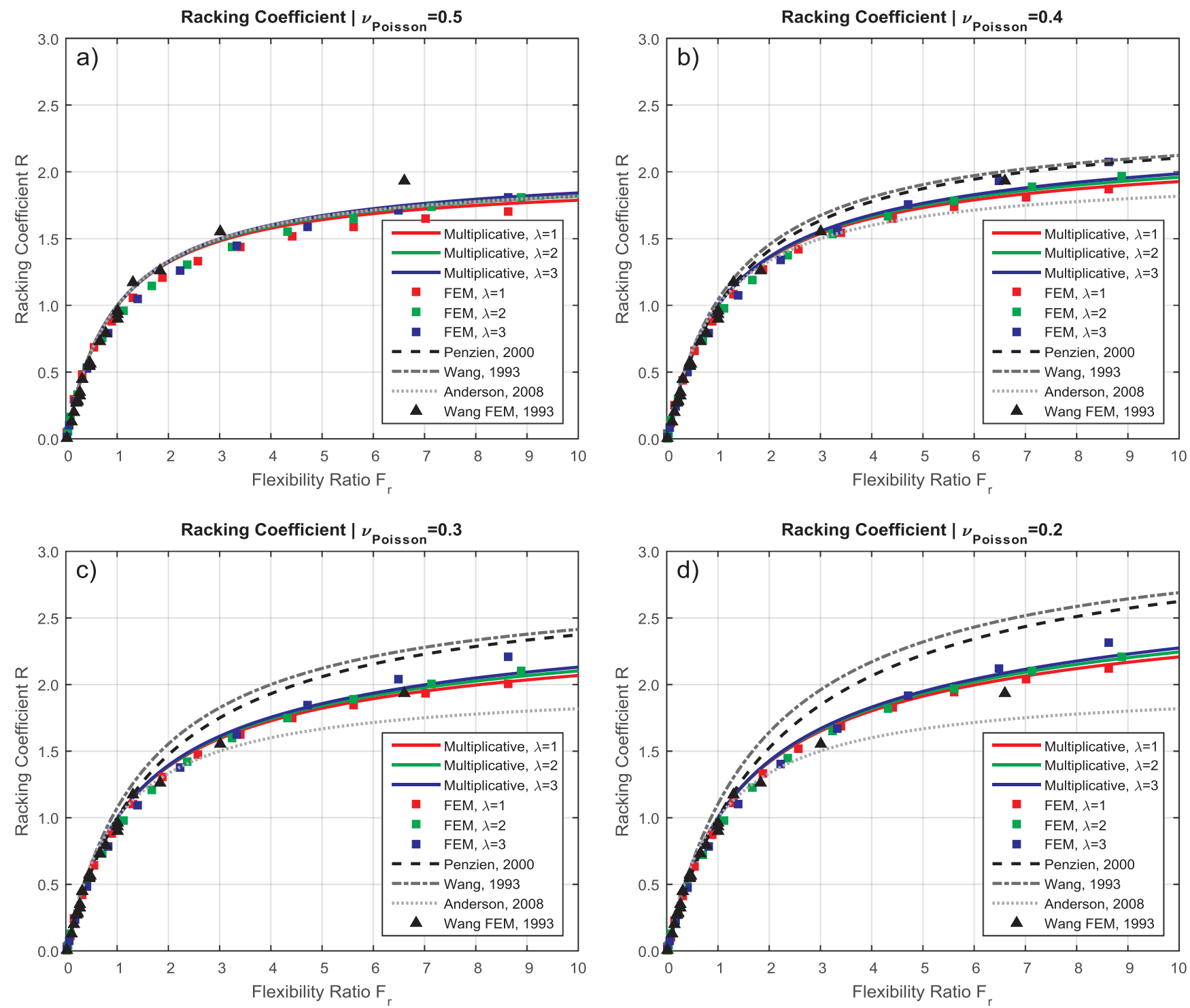

Fig. 6. Comparison of closed-form solutions by Wang (1993), Penzien (2000), Anderson et al. (2008), and the ones proposed in this study, plotted along with the finite element results developed in this study, and the ones provided by Wang (1993). For Poisson coefficients of (a) $\nu=0.5$, (b) $\nu=0.4$, (c) $\nu=0.3$, (d) $\nu=0.2$.

a)

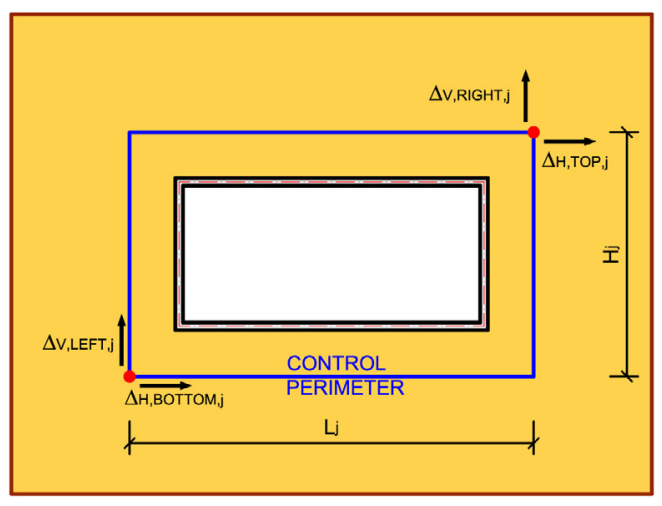

b)

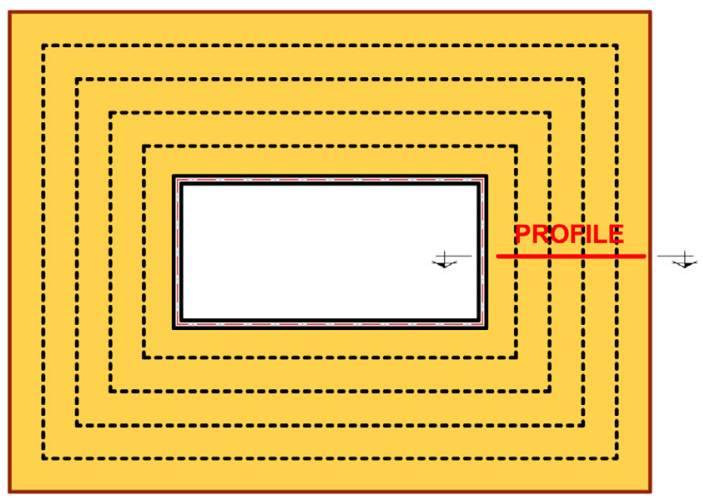

Fig. 7. (a) Displacement control points for an intermediate control perimeter $j$ of width $L_{j}$ and depth $H_{j}$, to compute intermediate racking coefficients. (b) Location of the racking coefficient profile. 

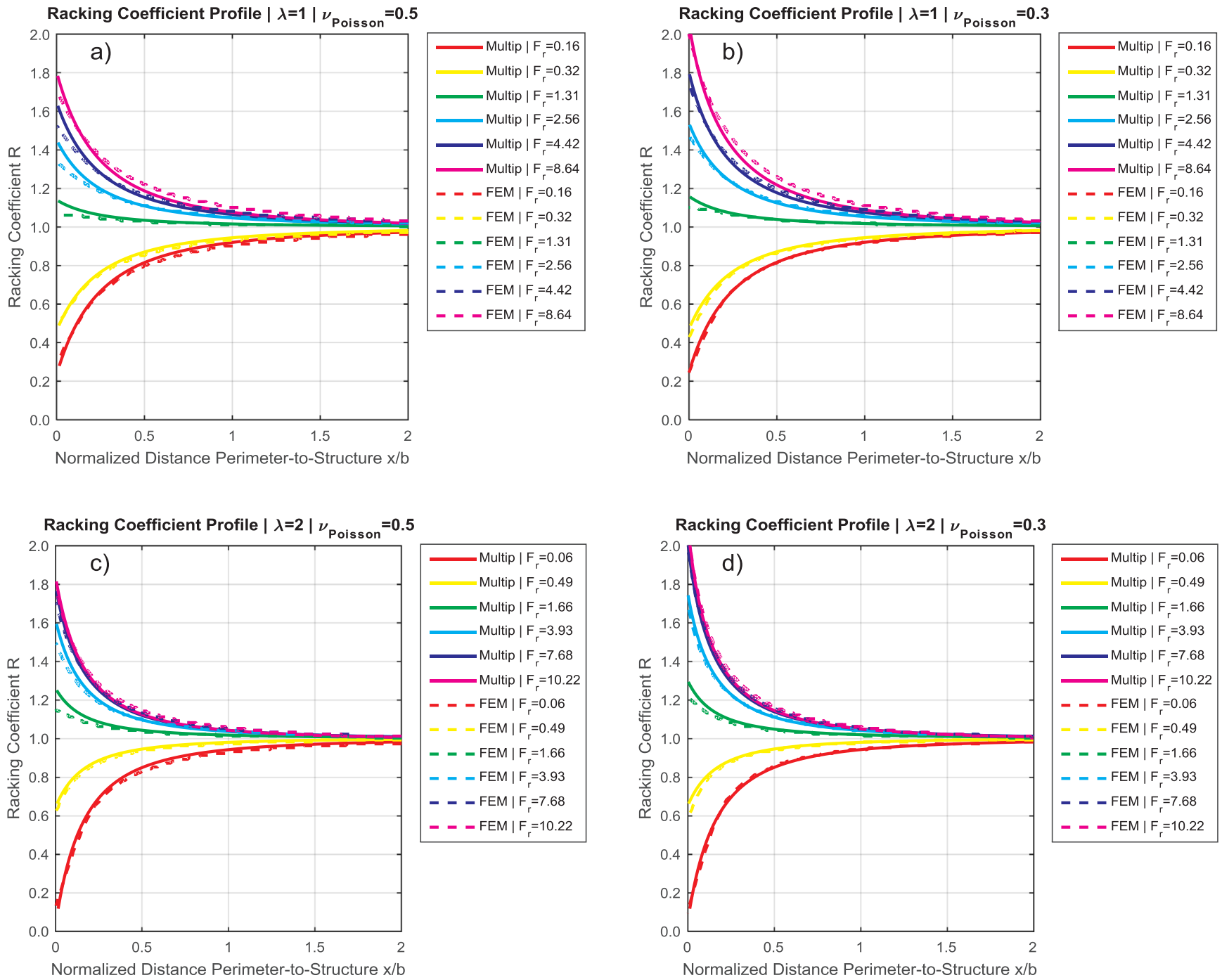

Fig. 8. Racking coefficient profiles computed with closed-form (solid lines), and FEM (dotted lines) for different aspect ratios $\lambda$, $F_{R}$ values, and Poisson coefficients. Horizontal distances range from adjacent to the structure $\left(X_{\text {PERIM }} / b_{\text {STRU }}=0\right)$ to a distance of 2 structure widths $\left(X_{\text {PERIM }} / b_{\text {STRU }}=2\right)$.

Tsinidis and Pitilakis, 2018), where color shades represent vertical displacements normalized to the maximum in each figure (not among figures) as to highlight the vertical displacement pattern in each figure.

When considering the displacement pattern of the block mechanism presented before, an explanation to this rocking behavior comes naturally. Referring to (Fig. 10), and considering for instance the case of a very stiff structure $\left(F_{R} \approx 0\right)$, the racking of the structure will be of a lesser value than the racking corresponding to the outer boundaries of a

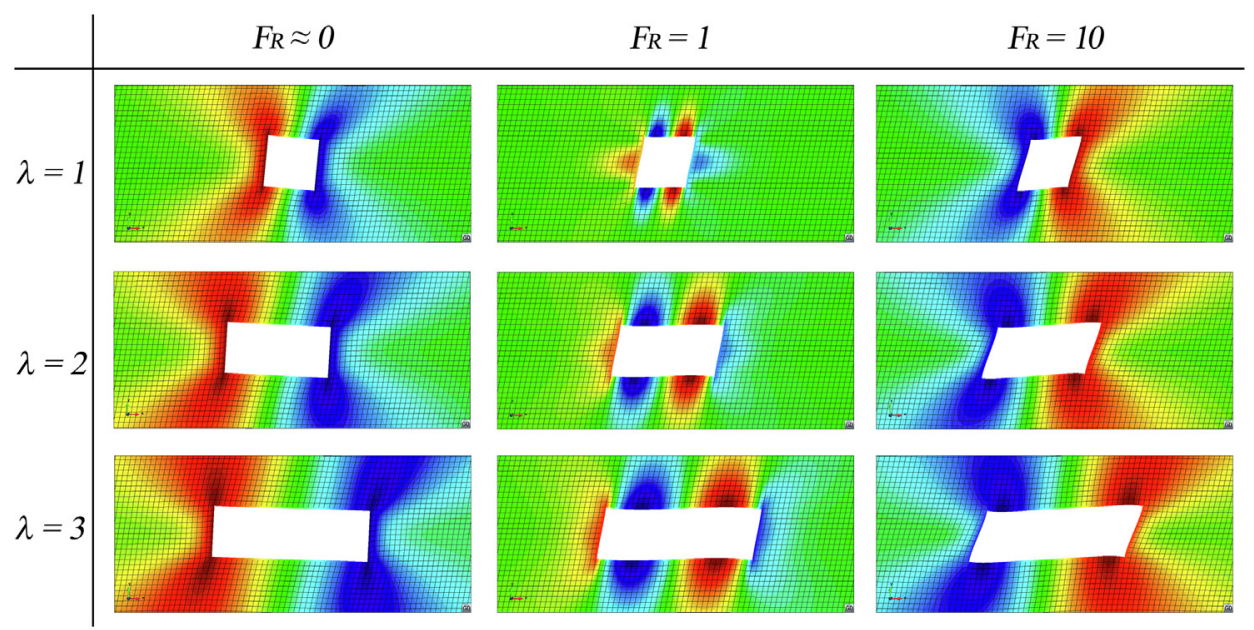

Fig. 9. Displacement patterns resulting from FEM analyses as a function of the flexibility ratio $F_{R}$ and aspect ratio $\lambda$. 


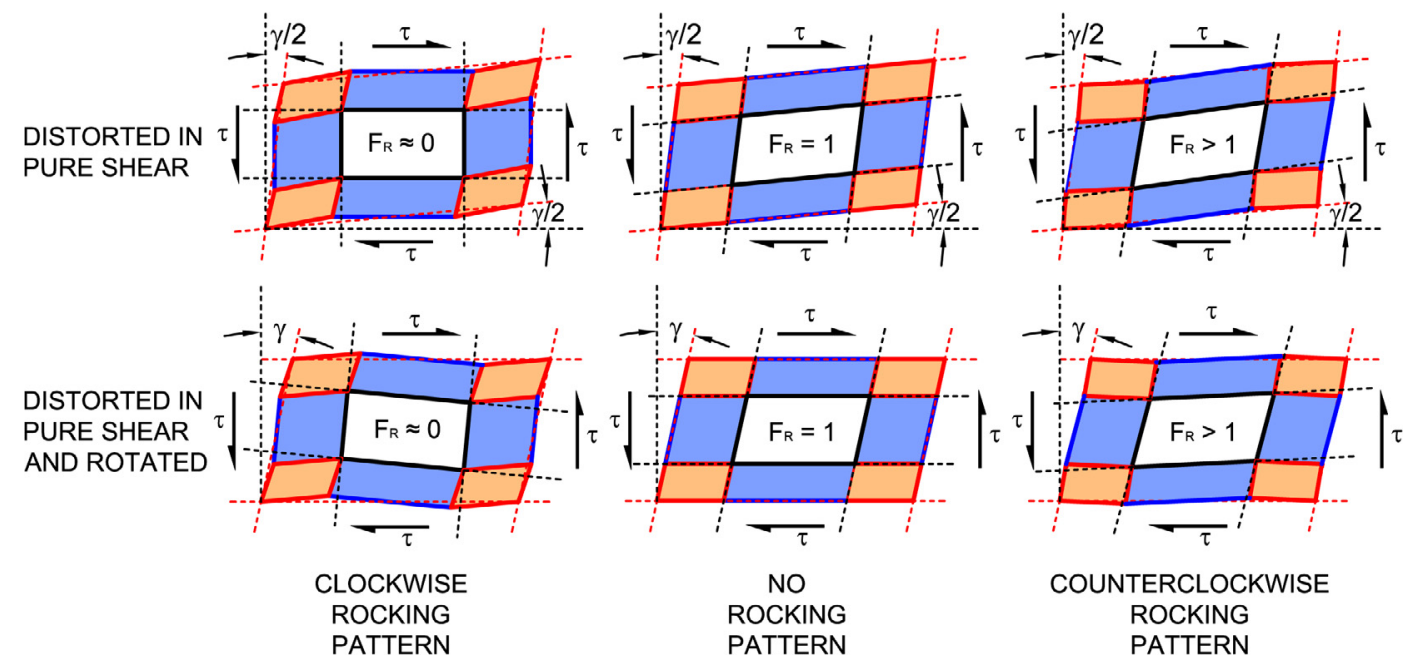

Fig. 10. Conceptual rocking patterns as a function of the structure stiffness.

given soil control perimeter. Therefore, when we consider the boundary conditions for the displacement field (i.e. the horizontal bottom boundary of the outermost soil perimeter must remain horizontal because of the contact with the bedrock), we must apply a rigid body rotation to the block ensemble to compatibilize prescribed displacements. For the particular case depicted of $F_{R} \approx 0$, this yields a clockwise rigid body rotation of the embedded structure if the outer soil distortion is rightwards.

If the structure is as stiff as the substituted soil $\left(F_{R}=1\right)$, the racking of the structure will be of the same magnitude as the outer soil perimeter, and when applying the rigid body rotation to comply with the boundary conditions, the structure slab will not show any rotation. Finally, if the structure is softer than the surrounding soil $\left(F_{R}>1\right)$, given that the racking of the structure will be larger than the racking corresponding to the outer boundaries of the soil block, the rigid body rotation applied to comply with the boundary conditions will not be enough to cancel the rotation of, say, the horizontal slabs. Therefore, a counterclockwise rotation will be apparent if the shearing is rightwards.

Considering the successive perimeters conceptual model proposed in this paper, the cumulative horizontal boundary rotation of each inner perimeter, relative to each adjacent outer perimeter would yield a total structure rotation in a direction depending on the $F_{R}$ value. Conceptually, we can think of this rocking behavior as a matter of perspective, which depends on the boundary that we are choosing as a reference and on which direction respective to this the structure deforms. Quantitatively, considering the block model depicted in previous sections, and following the notation in (Tsinidis, 2017), we can think that the relative rotation of the horizontal boundaries within two consecutive perimeters $i-1$ (inner) and $i$ (outer), normalized to the $i$ (outer) shearing deformation is given by Eq. (21).

$\tilde{\theta}_{i-1, i}=\frac{\gamma_{V, i-1}-\gamma_{V, i}}{\gamma_{H, i}+\gamma_{V, i}}$

Given that, when the surrounding soil at a far distance is strained in pure shear a magnitude $\gamma_{F F}$, the $i$ perimeter will deform its racking coefficient $R_{P E R I M i}$ times the free field strain $\gamma_{F F}$, the total angle rotated by the structure slab can be computed by adding all the relative angles rotated between consecutive perimeters $(i-1$, and $i$ ) normalized to the shear distortion of the outer perimeter $i$, weighting each relative angle by the racking coefficient of that outer perimeter $i$, as in Eq. (22).

$\frac{\theta}{\gamma_{F F}} \cong \sum_{i=1}^{i=n} \widetilde{\theta}_{i-1, i} \cdot R_{P E R I M i}=\sum_{i=1}^{n}\left(\frac{\gamma_{V, i-1}-\gamma_{V, i}}{\gamma_{H, i}+\gamma_{V, i}} \cdot \prod_{j=i+1}^{j=n} \frac{\gamma_{j-1}}{\gamma_{j}}\right)$

To assess the accuracy of the proposed expression, the results have been compared to a series of FEM analyses, where the rotation of the bottom slab has been computed as in Eq. (23), where $\Delta_{V E R T, R I G H T}$ and $\Delta_{V E R T, L E F T}$ stand for the vertical displacements of the top-right and bottom-left embedded structure corners, and $b$ stands for the structure width.

$\theta_{F E M}=\frac{\Delta_{V E R T, R I G H T}-\Delta_{V E R T, L E F T}}{b}$

The results of the proposed closed-form expression, and numerical results of the FEM analyses show a reasonably good agreement as shown in (Fig. 11).

\section{Modification of the proposed solutions for consideration of the Poisson coefficient}

As explained previously, the underlying mechanical model of the proposed solutions, namely the block behavior assumption, is unable to consider the dependence on the Poisson coefficient. In order to cater for this effect, a simple adjustment factor $\alpha$ can be introduced for the multiplicative closed-form racking expression given by Eq. (18), for the multiplicative closed-form racking profile expression given by Eq. (19), and for the summative closed-form rocking expression given by Eq. (22). This modification factor should be able to adjust the closed-form solution results to the finite element model results.

The chosen functional form for the modification factor $\alpha$ is shown in Eq. (24), being a logarithmic expression depending both on the Poisson coefficient $\nu$ and the flexibility ratio $F_{R}$. The corresponding coefficients computed by a best-fit approach are shown in Table 1 .

$\alpha=\left\{\begin{array}{c}1, F_{R}<1 \\ \left(\alpha_{1} \cdot v+\alpha_{2}\right) \cdot \operatorname{Ln}\left(F_{R}\right)+\alpha_{3}, F_{R} \geq 1\end{array}\right.$

After application of the modification factor $\alpha$, the closed form expression for the racking coefficient becomes Eq. (25), and where the term $\widetilde{R_{i}}$ is given by Eq. (17).

$R=\frac{\gamma_{\text {STRU }}}{\gamma_{F F}} \cong \alpha \cdot \prod_{i=1}^{i=n} \tilde{R_{i}}$

Similarly, after application of the modification factor $\alpha$, the closed form expression for the racking coefficient profile, at control perimeter $j$, becomes Eq. (26). In the computation of the $\alpha_{j}$ factor, the value of the flexibility ratio $F_{R}$ should be that of the $j$ perimeter being analyzed.

$R_{P E R I M j}=\frac{\gamma_{P E R I M j}}{\gamma_{F F}} \cong \alpha_{j} \cdot \prod_{i=j+1}^{i=n} \widetilde{R}_{i}$ 

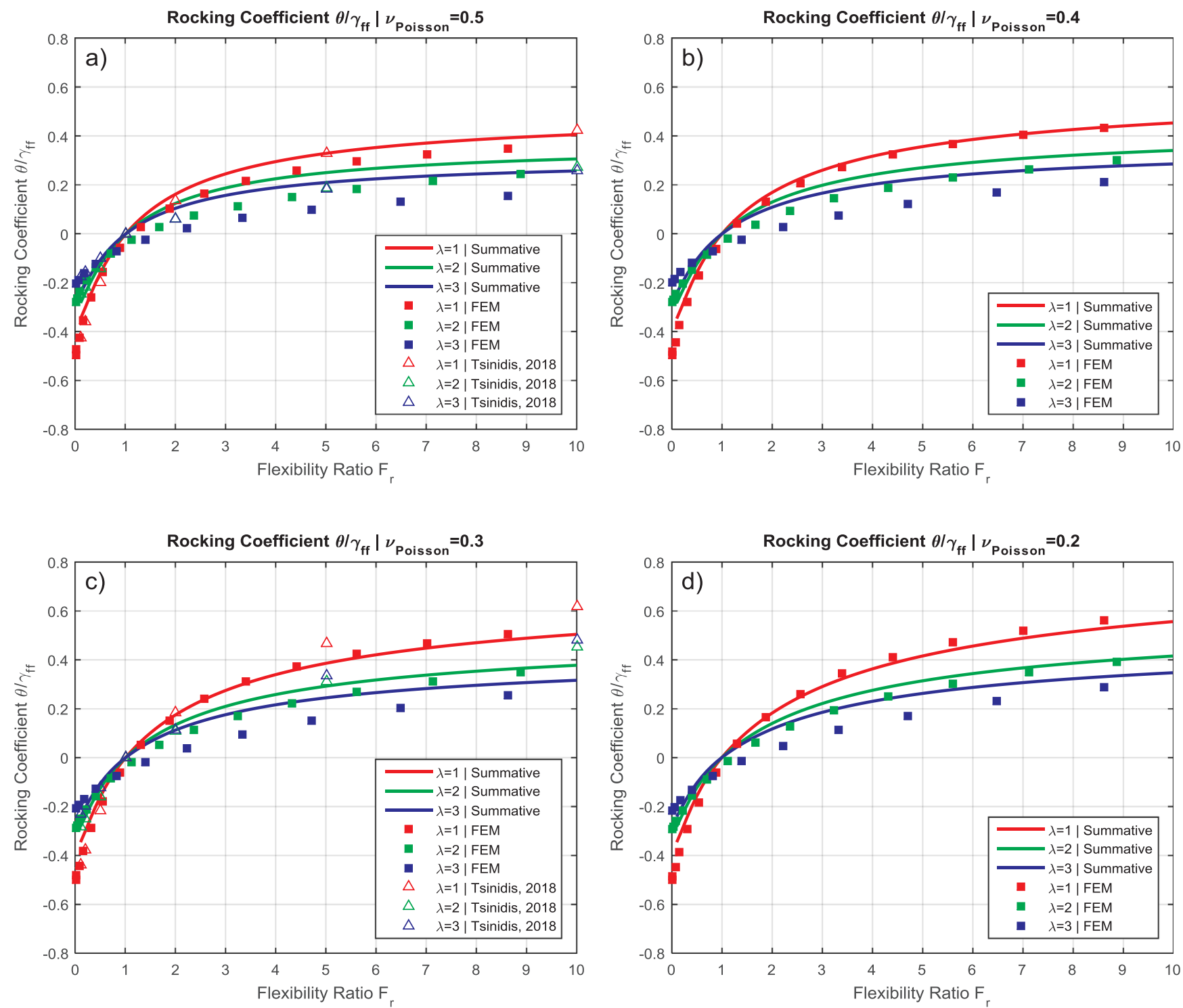

Fig. 11. Comparison of closed-form solutions for the rocking coefficient for three aspect ratios $\lambda=1,2,3$, and FEM results developed in this study, and those in (Tsinidis and Pitilakis, 2018), where available. For Poisson coefficients of (a) $\nu=0.5$, (b) $\nu=0.4$, (c) $\nu=0.3$, (d) $\nu=0.2$.

Table 1

Coefficients for the modification factor $\alpha$.

\begin{tabular}{lll}
\hline$\alpha_{1}$ & $\alpha_{2}$ & $\alpha_{3}$ \\
\hline-0.342 & 0.176 & 1.0 \\
\hline
\end{tabular}

Lastly, after the application of the modification factor $\alpha$, the closed form expression for the rocking coefficient becomes Eq. (27). As in the previous case, in the computation of the $\alpha_{i}$ factor, the value of the flexibility ratio $F_{R}$ should be that of the $i$ perimeter being evaluated.

$\frac{\theta}{\gamma_{F F}} \cong \sum_{i=1}^{n}\left(\alpha_{i} \cdot \tilde{\theta}_{i-1, i}\right) \cdot\left(\alpha_{i} \cdot R_{\text {PERIMi }}\right)=\sum_{i=1}^{n}\left(\left(\alpha_{i} \cdot \frac{\gamma_{V, i-1}-\gamma_{V, i}}{\gamma_{H, i}+\gamma_{V, i}}\right) \cdot\left(\alpha_{i} \cdot \prod_{j=i+1}^{j=n} \frac{\gamma_{j-1}}{\gamma_{j}}\right)\right)$

\section{Performance of the proposed closed-form solutions}

It is insightful to examine the performance of the closed-form solutions, both in terms of efficiency respective to the fineness of the discretization (i.e. number of control perimeters involved in the closed- form expressions), and accuracy respective to the results obtained with the FEM analyses. A convergence ratio dependent on the number of control perimeters involved in the closed-form solution can be computed as the ratio of the closed-form racking coefficient (evaluated by means of Eq. (25)) to the racking coefficient obtained with the FEM analyses for the case of $\nu=0.5$ and $\lambda=1$. Corresponding results for a range of flexibility ratios $F_{R}$ are shown in (Fig. 12a), where it can be appreciated that for approximately 200 perimeters an accuracy of $\pm 10 \%$ is reached. A further increase in the number of perimeters provides stability to the predicted racking coefficient value.

The accuracy of the proposed racking and rocking coefficients solutions, computed as the ratio of the values obtained with the closedforms to the FEM analyses, has been evaluated and shown in (Fig. 12b) for a range of flexibility ratios $F_{R}$, aspect ratios $\lambda$, and Poisson coefficients $\nu$. As it can be appreciated, the racking coefficient provides a good accuracy in the order of $\pm 10 \%$, while the rocking coefficient shows a larger dispersion.

\section{Summary and conclusions}

A new and intuitive method has been presented to analyze the shear 

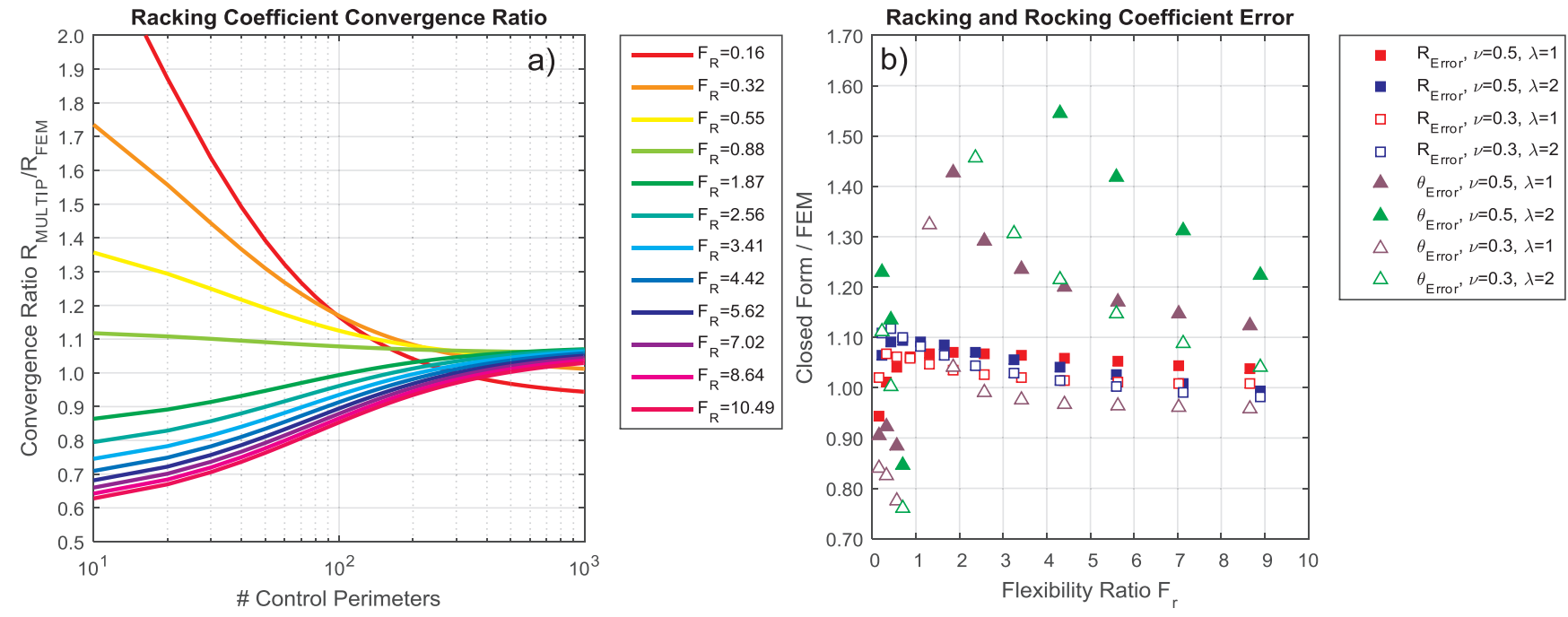

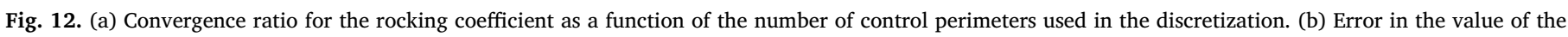
racking and rocking coefficients computed with the closed-form solutions respective to the value computed with the FEM analyses.

distortion of underground rectangular structures subjected to far field shear strains. The method is applicable for the case of a homogeneous, isotropic, elastic medium, and elastic structure, buried at a sufficient depth as to be able to assume that the medium is subjected to a pure shear state.

The method is based on first principles of mechanics, namely on the simple behavior of elastic blocks subjected to pure shear, and on the discretization of the soil-structure ensemble in successive control perimeters to which recursively apply the simple concept of a shear block.

This method is able to accurately predict three magnitudes: the racking distortion of the structure, the rocking rotation of the structure, and the soil shear distortion profile near and far from the structure boundaries. Closed-form expressions are provided for these three magnitudes. This method has been verified by comparing its predictions with those obtained with numerical analyses by finite element models, and the numerical results and closed form solutions of other researchers.

The method can be easily implemented in a spreadsheet, but more importantly, it provides a simple conceptual framework to understand the mechanic behavior of a rectangular structure embedded in a surrounding medium subjected to a far field pure shear deformation.

\section{Appendix A. Supplementary material}

Supplementary data to this article can be found online at https:// doi.org/10.1016/j.tust.2019.03.002.

\section{References}

Abate, G., Massimino, M.R., 2017. Parametric analysis of the seismic response of coupled tunnel-soil-aboveground building systems by numerical modelling. Bull. Earthq Eng. 15, 443-467. https://doi.org/10.1007/s10518-016-9975-7.

Anderson, D.G., Martin, G.R., Lam, I., Wang, J.N., 2008. NCHRP 611 - Seismic Analysis and design of Retaining Walls, Buried Structures, Slopes, and Embankments. Washington, DC.

Bobet, A., 2010. Drained and undrained response of deep tunnels subjected to far-field shear loading. Tunn. Undergr. Sp. Technol. 25, 21-31. https://doi.org/10.1016/j. tust.2009.08.001.

Bobet, A., Fernandez, G., Huo, H., Ramirez, J., 2008. A practical iterative procedure to estimate seismic-induced deformations of shallow rectangular structures. Can. Geotech. J. 45, 923-938. https://doi.org/10.1139/T08-026.

Cilingir, U., Madabhushi, S.P.G., 2011. A model study on the effects of input motion on the seismic behaviour of tunnels. Soil Dyn. Earthq. Eng. 31, 452-462. https://doi. org/10.1016/j.soildyn.2010.10.004.

Debiasi, E., Gajo, A., Zonta, D., 2013. On the seismic response of shallow-buried rectangular structures. Tunn. Undergr. Sp. Technol. 38, 99-113.

Fabozzi, S., Licata, V., Autuori, S., Bilotta, E., Russo, G., Silvestri, F., 2017. Prediction of the seismic behavior of an underground railway station and a tunnel in Napoli (Italy).
Undergr. Sp. 2, 88-105. https://doi.org/10.1016/j.undsp.2017.03.005.

Guoxing, C., Su, C., Xi, Z., Xiuli, D., Chengzhi, Q.I., Zhihua, W., 2015. Shaking-table tests and numerical simulations on a subway structure in soft soil. Soil Dyn. Earthq. Eng. 76, 13-28. https://doi.org/10.1016/j.soildyn.2014.12.012.

Hashash, Y.M.A., Hook, J.J., Schmidt, B., I-Chiang Yao, J., 2001. Seismic design and analysis of underground structures. Tunn. Undergr. Sp. Technol. 16, 247-293. https://doi.org/10.1016/S0886-7798(01)00051-7.

Hashash, Y.M.A., Karina, K., Koutsoftas, D., O'Riordan, N., 2010. Seismic design considerations for underground box structures. In: Proceedings of the 2010 Earth Retention Conference, pp. 620-637. https://doi.org/10.1061/41128(384)64.

Hendron, A.J., Fernandez, G., 1983. Dynamic and static design considerations for underground chambers. In: Howard, T.R. (Ed.), Seismic Design of Embankments and Caverns. ASCE, New York, pp. 157-197.

Hudson, J.A., Harrison, J.P., 2000. Engineering Rock Mechanics: An Introduction to the Principles. Elsevier.

Hung, C.J., Monsees, J.E., Munfah, N., Wisniewski, J., 2009. Technical Manual for Design and Construction of Road Tunnels - Civil Elements. Washington, D.C.

Huo, H., Bobet, A., Fernández, G., Ramírez, J., 2005. Load transfer mechanisms between underground structure and surrounding ground: evaluation of the failure of the Daikai station. J. Geotech. Geoenviron. Eng. 131, 1522-1534.

Huo, H., Bobet, A., Fernández, G., Ramírez, J., 2006. Analytical solution for deep rectangular structures subjected to far-field shear stresses. Tunn. Undergr. Sp. Technol. 21, 613-625. https://doi.org/10.1016/j.tust.2005.12.135.

Iwatate, T., Kobayashi, Y., Kusu, H., Rin, K., 2000. Investigation and shaking table tests of subway structures of the Hyogoken-Nanbu earthquake. In: Proceedings 12th World Conference on Earthquake Engineering. Auckland, New Zealand, pp. 1-8.

Kuesel, T.B., 1969. Earthquake design criteria for subways. J. Struct. Div. ASCE 95, 6616-6626.

LA Metro Authority, 2012. Metro Rail Design Criteria Section 5 Structural/Geotechnical. Los Angeles, CA.

McKenna, F., Scott, M.H., Fenves, G.L., 2010. Nonlinear finite-element analysis software architecture using object composition. J. Comput. Civ. Eng. 24, 95-107. https://doi. org /10.1061/(ASCE)CP.1943-5487.0000002.

Merrit, J.L., Monsees, J.E., Hendron, A.J., 1985. Seismic design of underground structures. In: In: Mann, C.D., Kelley, M.N. (Eds.), Proc. of the 1985 Rapid Excavation Tunnelling Conference, Society of Mining, Metallurgical and Petroleum Engineers 1. New York, pp. 104-131.

Monsees, J.E., 1991. Underground seismic design. In: Fall Lecture Series of the Boston Society of Civil Engineers Section. Structural Group Lecture Series, Boston, MA, pp $1-41$.

Peck, R.B., 1969. Deep excavations and tunneling in soft ground. In: 7th International Conference on Soil Mechanics and Foundation Engineering, pp. 225-290.

Penzien, J., 2000. Seismically induced racking of tunnel linings. Earthq. Eng. Struct. Dyn. 29, 683-691. https://doi.org/10.1002/(SICI)1096-9845(200005)29:5 < 683::AIDEQE932 > 3.0.CO;2-1.

Penzien, J., Wu, C.L., 1998. Stresses in linings of bored tunnels. Earthq. Eng. Struct. Dyn. 27, 283-300. https://doi.org/10.1002/(SICI)1096-9845(199803)27:3<283::AIDEQE732 > 3.0.CO;2-T.

Pitilakis, K., Anastasiadis, A., Raptakis, D., Boussolas, N., Papageorgiou, E., 2007. Seismic design loads for metropolitan subway tunnels: the case of the thessaloniki Metro. In: Proceedings of the 4th International Conference on Earthquake Geotechnical Engineering. Thessaloniki, pp. 1-15.

Pitilakis, K., Tsinidis, G., 2016. Recent advances on the seismic behaviour and design of tunnels. In: Conference in Honour of Michele Maugeri. Catania, Italy, pp. 1-46.

Tsinidis, G., 2017. Response characteristics of rectangular tunnels in soft soil subjected to transversal ground shaking. Tunn. Undergr. Sp. Technol. 62, 1-22. https://doi.org/ 10.1016/j.tust.2016.11.003. 
Tsinidis, G., Pitilakis, K., 2018. Improved R-F relations for the transversal seismic analysis of rectangular tunnels. Soil Dyn. Earthq. Eng. 107, 48-65. https://doi.org/10.1016/j. soildyn.2018.01.004.

Tsinidis, G., Pitilakis, K., Anagnostopoulos, C., Madabhushi, G., 2015a. Seismic response and design of rectangular tunnels. In: Proceedings of the SECED 2015 Conference: Earthquake Risk and Engineering Towards a Resilient World. World, Cambridge, UK, pp. $1-10$.

Tsinidis, G., Pitilakis, K., Heron, C., 2015b. Dynamic response of flexible square tunnels: centrifuge testing and validation of existing design methodologies. Geotechnique 65, 401-417. https://doi.org/10.1680/geot.SIP.15.P.004.

Tsinidis, G., Pitilakis, K., Mantikas, G., 2016. Deformation patterns of rectangular tunnels in soft soils subjected to ground shaking. In: Proceedings of the 1st ICONHIC
International Conference on Natural Hazards \& Infrastructure. Chania, Greece, pp. 1-9.

Ulgen, D., Saglam, S., Ozkan, M.Y., 2015. Dynamic response of a flexible rectangular underground structure in sand: centrifuge modeling. Bull. Earthq. Eng. 13, 2547-2566. https://doi.org/10.1007/s10518-015-9736-z.

Wang, H.F., Lou, M.L., Chen, X., Zhai, Y.M., 2013. Structure-soil-structure interaction between underground structure and ground structure. Soil Dyn. Earthq. Eng. 54, 31-38. https://doi.org/10.1016/j.soildyn.2013.07.015.

Wang, J.N., 1993. Seismic Design of Tunnels. A Simple State of the Art Approach. New York, NY.

WSDOT, 2010. Appendix B8 Seismic Design Criteria SR 99 Bored Tunnel Alternative Design-Build Project. Seattle, WA. 